\title{
Anatomy and Vascular Biology of the Cells in the Portal Circulation
}

\author{
Massimo Pinzani, $M D, P h D$ \\ and Francesco Vizzutti, MD
}

\author{
Contents \\ INTRODUCTION \\ Anatomic Considerations of the Normal Portal Circulation \\ Biology of Portal Cells Including Sinusoidal Endothelial \\ Cells and HSCs \\ Major Signaling Pathways Relevant to Endothelial-Smooth \\ Muscle Cell Interactions \\ REFERENCES
}

\section{INTRODUCTION}

Portal hypertension occurring during the natural course of liver cirrhosis is a consequence of the increased intrahepatic resistance to portal flow. For a long time, this phenomenon has been ascribed only to the profound changes of liver tissue angioarchitecture consequent to the progression of the fibrogenic process. However, studies performed during the last decade have demonstrated that there is also an increased vascular tone that could be modulated to a certain extent by pharmacological agents. The aim of this chapter is to provide general information on the anatomy of the portal systems and on the regulation of vascular tone in this specific vascular district and in the splanchnic circulation. Information about the collateral circulation that becomes relevant in the case of portal hypertension is also provided.

In addition, because of the many studies performed in animal models and isolated and cultured hepatic cell, attention will be paid to the biology of these cells and to the relative pathophysiological implications. In particular, hepatic stellate cells, now regarded as liver-specific pericytes, are likely to play an important role in the progression of portal hypertension because of their active role in the deposition of fibrillar extracellular matrix and of their contractile properties. In this context, several vasoconstricting agonists, whose expression is increased in fibrotic liver, may play a role in inducing contraction 
of hepatic stellate cells as well as of other resident cells characterized by contractile ability. The features of different vasoactive agents will be analyzed and their potential involvement in physiological and pathological conditions thoroughly discussed.

\section{ANATOMIC CONSIDERATIONS OF THE NORMAL PORTAL CIRCULATION}

A portal venous system is defined as one beginning and ending in capillaries. The name "portal vein" derives from the notion that it is the gate into which the splanchnic circulatory system is connected to the liver (porta $=$ gate). The name portal vein is applied to the venous system that originates in the capillaries of the intestine and terminates in the hepatic sinusoids. Nutrients absorbed from the gastrointestinal tract, in addition to hormones such as glucagons and insulin released by the pancreas, are directly delivered to the liver in high concentrations.

\section{Embryology of the Portal System}

The portal venous system originates from the two vitelline and the two umbilical veins. The vitelline veins, which drain blood from the yolk sac, intercommunicate in the septum trasversum, at which point the liver sinusoids and lobules develop. The extrahepatic portal system develops primarily from the left vitelline vein (which is later joined by the splenic vein to form the portal vein), whereas the intrahepatic portal circulation originates from the umbilical veins. In addition, the left umbilical vein communicates with the venous sinus connecting with the inferior vena cava, thus allowing a large quantity of blood to bypass the liver in the fetal circulation. Soon after birth, the umbilical vein is obliterated and the normal adult circulation is established. Despite this complexity in the development of the portal system, only very few congenital anomalies of the portal venous system are observed.

\section{Gross Anatomy of the Portal System}

The portal vein is a vessel collecting the venous blood of the abdominal part of the alimentary tract, spleen, pancreas, and gallbladder to the liver. The portal vein begins at the level of the second lumbar vertebra, just behind the neck of the pancreas as an upward continuation of the superior mesenteric vein after this vessel has been joined by the splenic vein. The superior mesenteric vein $(0.78 \mathrm{~cm}$ in diameter) is primarily formed by all the veins draining the small bowel, with significant further contributions of the ileocolic, right colic, and middle colic veins. It runs in the root of the mesentery, in front of the third portion of the duodenum to merge with the splenic vein. The splenic vein $(0.94 \mathrm{~cm}$ in diameter) originates with five to six branches that return the blood from the spleen and unite to form a single nontortuous vessel at the splenic hilum and join near the tail of the pancreas with the short gastric vessels to form the main splenic vein. This vein proceeds transversally, close to the hilum of the left kidney, in the body and head of the pancreas, receiving numerous tributaries from this latter portion of the pancreas. The left gastroepiploic vein joins the splenic vein near the spleen, and the inferior mesenteric vein $(0.24 \mathrm{~cm}$ diameter), collecting blood from the left part of the colon and rectum, usually enters its middle third. Occasionally (one-third of subjects) the inferior mesenteric vein enters directly into the superior mesenteric vein or at its junction with the splenic vein. On its way to the porta hepatis, the portal vein trunk receives (in some variants) the superior pancreaticoduodenal 
vein (with right gastroepiploic vein) and the right gastric (pyloric) veins. The left gastric (coronary) vein joins the portal vein at its origin $50 \%$ of the time, and it joins the splenic instead of the portal vein in the other $50 \%$ of subjects. Coronary vein runs upward along the lesser curvature of the stomach, where it receives some esophageal veins.

The portal system carries all the blood from the alimentary tract to the liver and, thus, in the normal subject all of the above-named veins have blood flow directed toward the liver. The segment of the portal vein after the last afferent branch runs in the hepatoduodenal ligaments (the free edge of the lesser omentum) in a plane dorsal to the bile ducts and the hepatic artery. This segment extends for approx $6-8 \mathrm{~cm}$ before entering the liver and it is $1-1.2 \mathrm{~cm}$ in diameter. The portal vein is not provided with valves, so the pressure is transmitted freely back to the afferent branches. The portal vein pressure normally ranges between 5 and $10 \mathrm{mmHg}$ (depending on the method of measurement). Normal fasting hepatic blood flow is approx $1500 \mathrm{~mL} / \mathrm{min}$. The best available estimates in humans indicate that about two-thirds of the total hepatic blood flow and about one-half of the oxygen consumption are supplied by the portal vein, whereas the remainder is supplied by the hepatic artery. This dual hepatic blood supply makes the liver rather resistant to hypoxia. Accordingly, ligation of the portal vein does not cause hepatocellular necrosis. Similarly, accidental ligation of the hepatic artery or its major branches does not necessarily lead to hepatic failure. The portal trunk divides into two lobar veins before entering the portal fissure. The right lobar branch, short and thick, then receives the cystic vein. The left lobar vein is longer than the right and consists of a transverse and an umbilical part. The latter is the remainder of the umbilical parts. The recanalized umbilical or paraumbilical veins arise from the umbilical portion of the left portal vein and pass through the round ligament to the anterior abdominal wall, where they may become evident, in the presence of portal hypertension, in the umbilical varices.

According to the distribution of major portal vein branches, so-called segmental branches, the liver can be divided into functional segments. Each segment depends on its major vessel for blood supply. The right branch of the portal vein is usually less than $3 \mathrm{~cm}$ long and runs more vertically. It divides into anterior and posterior branches, which supply the anterior and posterior parts of the right lobe. Each of these vessels divides again into superior and inferior branches. The left lobar vein gives branches to the quadrate lobe and to the caudate lobe, before entering the parenchyma at the left end of the porta hepatis. A separate branch may arise near the bifurcation to supply the caudate lobe. The vein is then joined by the obliterated umbilical vein as it turns medially. The terminal part of the vessel continues into segment IV, which it supplies with ascending and descending branches. In addition to the main portal vein and its branches, the liver receives other veins from the splanchnic circulation, the so-called parabiliary venous system of Couinaud. This highly variable plexus includes several veins that arise from the pancreaticoduodenal or pyloric veins and drain into the portal vein or directly into hepatic segments, especially segment IV. This plexus provides examples of the metabolic effects of proximity to an insulin source. Veins arising from the pancreatic region would carry blood with high insulin levels and pyloric veins would carry low-insulin blood. The anatomy of these veins could explain some examples of focal fatty liver and focal fatty sparing, in fact, insulin determines the ability of the liver to accumulate triglycerides (1).

The other vessel supplying the liver is the hepatic artery. About one-third of the total hepatic blood flow is supplied by the hepatic artery. The common hepatic artery is the second major branch of the celiac axis. It runs to the right along the upper border of the 
pancreas in the context of the right gastropancreatic fold, which conducts the artery to the medial border of the hepatoduodenal part of the lesser omentum. It ascends in front of the portal vein in $91 \%$ of subjects and to the left of and behind the bile duct in $64 \%$ of cases. It divides into the left and the right hepatic arteries to supply the corresponding hemilivers. Although the left and right hepatic arteries are end-arteries, they often anastomose within the hilar tissue (2). The right and left hepatic arteries each divide into two arteries that supply the right anterior and posterior sections and the left medial lateral sections, respectively. Another branch, the middle hepatic artery, arises from the left or right hepatic artery and supplies the quadrate lobe. The cystic artery arises from the right hepatic artery in the upper part of the Calot triangle (formed by the cystic duct, common hepatic duct, and inferior surface of liver) (3).

\section{Portal Collateral Circulation}

The portal system has numerous collaterals that interconnect with the systemic circulation. When portal pressure rises above $10 \mathrm{mmHg}$ potential portosystemic collaterals may develop. Formation of collaterals is a complex process involving the opening, dilation, and hypertrophy of preexisting vascular channels. It is possible that active neoangiogenesis is involved in the formation collateral vessels (4). The sites for the development of portal collateral vessels are those areas where veins draining into the portal system are in immediate juxtaposition to veins draining into the superior or inferior vena cava. Collaterals vessels could be classified into tree embryological groups: (1) junction of absorp-tive and protective epithelium (gastroesophageal and hemorrhoidal plexuses); (2) obliterated fetal circulation (umbilical or paraumbilical veins in round and falciform ligaments); and (3) organs derived from the gastrointestinal tract that became retroperitoneal or adhere to the abdominal wall because of pathologic process (portorenal plexus, veins of Retzius, surgical stomata, and other interventions connecting portal bed with the ascending lumbar azygos, renal, and adrenal veins).

The most important sites for the development of portosystemic collateral vessels are: (1) esophageal submucosal veins, supplied by the left gastric vein and draining into the superior vena cava through the azygos vein; (2) paraumbilical veins, although normally nonfunctional, can serve as an anastomosis between the umbilical part of the left portal vein and the hepigastric veins of the anterior abdominal wall that drain into the superior or inferior vena cava, and in special circumstances may form caput medusae at the umbilicus (Cruveilhier-Baumgarten syndrome); (3) rectal submucosal veins, supplied by the inferior mesenteric vein through the superior rectal vein and draining into the internal iliac veins through the middle rectal vein; (4) splenorenal shunts, in this case venous blood may be carried to left renal vein, either directly or by way of the diaphragmatic, pancreatic, or gastric veins; (5) short gastric veins communicate with the esophageal plexus. Moreover, within the cirrhotic liver, there is significant collateral flow in small veins that connect branches of the portal and hepatic veins (5).

\section{The Gastroesophageal Junction}

The normal venous anatomy of the gastroesophageal junction and of the lower esophagus is particularly relevant to this introductory chapter. Studies of Vianna et al. documented four distinct zones of esophageal venous drainage (from distal to proximal): (1) the gastric zone, which extends for $2-3 \mathrm{~cm}$ just below the gastroesophageal junction. This is the junctional zone between the stomach and lower oesophagus. Veins from this zone 
drain into the short gastric and left gastric veins. (2) The palisade zone extends $2-3 \mathrm{~cm}$ superiorly from the gastric zone into the lower esophagus and represent the watershed between the portal and systemic circulation. (3) The perforating or transitional zone extends approx $2 \mathrm{~cm}$ further up the esophagus above the palisade zone. Here, the organized longitudinal structure is lost, with veins looping and forming a network. The main feature of this zone is represented by the presence of perforating veins through the muscle wall of the esophagus linking the submucosal and paraesophageal venous plexuses that are tributaries of the azygos venous system. These perforating veins run circumferentially around the esophageal wall. In portal hypertensive patients, dilated perforating veins become incompetent and allow retrograde blood flow from the paraesophageal to the submucosal veins. This associated with the turbulent flow caused by pressure changes as a result of the respiratory movements, coughing and stretching may contribute to formation and dilation of varices. (4) The truncal zone is $8-10 \mathrm{~cm}$ long and is characterized by four of five longitudinal veins in the lamina propria. In this zone, perforating veins penetrate from the submucosa at irregular intervals to the external esophageal venous plexus.

In summary, venous drainage from the gastric fundus and the lesser curvature is directed inferiorly to the portal vein. In the palisade zone, there is to/from flow that is probably respiration dependent. The perforating veins connect the intrinsic and extrinsic esophageal plexuses. Flow in the truncal zone is inferior to the perforating zone. In conclusion, the perforating, transitional zone is the "critical area" for variceal rupture. Indeed, varices tend to be bigger and to form "nodules" at the distal end of the esophagus, at the level of the perforating veins (6).

\section{Structure and Function of the Splanchnic Vasculature}

The splanchnic circulation consists of those vascular beds perfused by the celiac, superior and inferior mesenteric arteries, and the portal vein. The organs perfused by the splanchnic vasculature receive about $25 \%$ of cardiac output and account for about $30 \%$ of total body oxygen consumption under resting conditions. Functional and/or structural changes in arterioles, capillaries, and venules can initiate or perpetuate an elevated portal pressure (e.g., dilation of arterioles, passive occlusion of capillaries, and active constriction of hepatic venules). The structural and functional characteristics of the microvasculature of the stomach and small and large intestine are very different from those of the liver. First, splanchnic capillaries are much less porous than the hepatic sinusoids and have a well-defined basement membrane. Although most splanchnic capillaries are fenestrated, the estimated pore size, 3.7 to $12 \mathrm{~nm}$ in radius, is between 50 and 100 times lower than that of the hepatic sinusoids. A very little amount of the total protein oncotic pressure may pass across a splanchnic capillary membrane; consequently, any increase in filtration in the splanchnic capillaries is quickly counterbalanced by an increase in the oncotic pressure difference between capillary lumen and interstitial space. In addition, there is evidence that the intestinal microvasculature autoregulates the capillary pressure and capillary filtration coefficient. There are significant differences between the intestinal and hepatic interstitium in terms of compliance; in fact, considerable interstitial fluid can accumulate without causing any major changes in interstitial pressure. Moreover, the intestines have a very efficient lymphatic system to remove interstitial edema. In normal conditions, approx $20 \%$ of the fluid absorbed by the small intestine is carried out to the general circulation by the lymphatics (7). 
Under basal conditions, splanchnic arterioles are partially constricted, and have the capacity to either further constrict or dilate. This arteriolar smooth muscle tone is the sum of multiple factors that tend to either relax or constrict vascular smooth muscle. A variety of metabolic end-products (e.g., adenosine), some endothelium-derived substances (e.g., nitric oxide), and certain neurotransmitters (e.g., acetylcholine) are known to relax arteriolar smooth muscle and produce vasodilation. Important vasoconstrictors influences on splanchnic arterioles include some circulating agents (e.g., angiotensin II), certain endothelium-derived substances (e.g., endothelin), and some neurotransmitters (norepinephrine). These factors can alter the contractile state of arteriolar smooth muscle either by acting directly on vascular smooth muscle (e.g., metabolic mediators) or by stimulating endothelial cells to release vasoactive agents that act on the underlying adjacent vascular smooth muscle (e.g., acetylcholine). Hypoxia, in terms of reduced oxygen delivery or increased oxygen demand, can lead to changes in arteriolar tone and consequent changes in blood flow. This effect appears to be mediated by terminal products of oxidative metabolism, such as adenosine, and tissue oxygen tension $\left(p \mathrm{O}_{2}\right)$ and appear to be one of the principal mechanisms of postprandial hyperemia. In fact, when tissue $p \mathrm{O}_{2}$ falls or extracellular adenosine concentration rises, arterioles dilate. Normally, splanchnic arterioles are exquisitely sensitive to acute changes in intravascular pressure. Vascular smooth muscle of splanchnic arterioles contracts intensely in response to stretch (inducing a sudden elevation in portal pressure). The intense dilation of arterioles observed in chronic portal hypertension likely reflects the accumulation of vasodilators [e.g., increased nitric oxide (NO) production, increased blood levels of glucagons] that overcome intrinsic myogenic vasoconstrictor factors (8).

Norepinephrine, angiotensin II, and vasopressin are estimated to account for more than two-thirds of basal splanchnic vascular tone. Norepinephrine generally elicits a profound, yet transient, reduction in splanchnic blood flow. Increased tissue levels of adenosine during vasoconstriction-mediated arterioles escape from norepinephrine-mediated vasoconstriction. On the contrary, vasopressin and angiotensin II cause a sustained reduction in splanchnic blood flow. Glucagon attenuates the splanchnic vasoconstrictive response induced by catecholamines, vasopressin, and angiotensin II through a downregulation of receptors and/or postreceptor mechanisms such as impairment of second-messenger activation in splanchnic vascular smooth muscle. A wide variety of hormones and peptides produced within the alimentary tract are capable of altering splanchnic blood flow when infused into arterial blood. Somatostatin and neuropeptide-Y are locally produced peptides that exert potent vasoconstrictor actions. Vasoactive intestinal polypeptide, substance $\mathrm{P}$, cholecystokinin, and gastrin are examples of gastrointestinal peptides that dilate splanchnic arterioles and increase blood flow.

Splanchnic organs exhibit an intrinsic ability to regulate local blood flow by modulating the tone of arterioles. Two examples of intrinsic vasoregulation are pressure-flow autoregulation and functional (postprandial) hyperemia. Pressure-flow autoregulation is the ability of an organ to maintain its constant blood flow when arterial pressure is reduced. This regulatory mechanism depends on metabolic or myogenic-mediated dilation of arterioles at lower intravascular pressures. However, pressure-flow autoregulation of splanchnic organs is not as potent and precise as in other vascular beds such as the heart, the brain, and the kidneys. Nevertheless, this autoregulation is improved in the postprandial phase (increased metabolic demand), when arterioles become more sensible to reductions in arterial pressure. Postprandial hyperemia has recently received much attention as a 
potential cause of rapid elevation in splanchnic blood flow and portal pressure that may lead to variceal formation, dilation, and explosion $(9,10)$. Splanchnic vasodilation and hyperemia is caused by the interaction of intrinsic (change in arteriolar transmural pressure and/or increase in vasodilator tissue metabolites) or extrinsic mechanisms (autonomic nervous system especially noncholinergic vagal reflexes) and the effect of nitric oxide, gastrointestinal hormones and peptides (gastrin, cholecystokinin and glucagons), autacoids (histamine, serotonin), osmolality, and prostaglandins. The relative contribution of these different factors is influenced by the composition of the meal (i.e., long-chain fatty acids appear to be the most potent stimulus) and the preprandial metabolic status of the affected organ.

\section{Nerves}

The liver is predominantly innervated by two plexuses, the anterior and the posterior, which communicate with each other. The anterior plexus surrounds the hepatic artery and is made up of fibers from the celiac ganglia and anterior vagus nerve. The posterior plexus surrounds the portal vein and bile duct and is formed from branches of the right celiac ganglia and posterior vagus. The vast majority of nerve fibers terminate in plexuses in the adventitia around hepatic arterioles and venules. Small fibers from these plexuses then end on smooth muscle cells in the media of these vessels. Within the liver cell plate, the majority of nerve fibers are observed in periportal regions. Some of the nerve fibers terminate on endothelial cells in the smallest hepatic arterioles, near the space of Disse, on Kupffer cells, and on hepatic stellate cells (HSC).

Hepatic innervation can be distinguished in extrinsic and intrinsic. The extrinsic innervation of the liver is constituted by: (1) efferent sympathetic nerve fibers and parasympathetic nerve fibers; these play a role in regulating the metabolic load of hepatocytes, hemodynamic and biliary motility; (2) afferent fibers, which are thought to be involved in osmo- and chemoreception. At the hilus, amyelinic fibers from the anterior and posterior plexuses enter the liver mainly around the hepatic artery. The intrinsic innervation is composed of fibers (mostly adrenergic, but also cholinergic and peptidergic) mainly associated with vascular and biliary structures in the portal spaces (11). Certain fibers enter liver lobule where they form a network around hepatocytes and extend into the sinusoidal wall, sometimes reaching the centrilobular vein. Some neuropeptides have been identified, such as vasointestinal peptide, neuropeptide Y, substance P, glucagon, and calcitonin gene-related peptide. Stimulation of sympathetic fibers causes an increase in vascular resistance and a decrease in hepatic blood volume.

\section{The Hepatic Portal Tree}

Segmental branches of the portal vein split dichotomously into equal sized branches, constituting a tree of conducting vessels that terminate in venules having an inner diameter of about $400 \mu \mathrm{m}$. Each branch of the afferent vessels is essential for proper function because it supplies blood to a specific area. There are few, if any, anastomoses that could provide collateral circulation if a major branch is impaired. In other words, the first portion of the portal system is merely conductive up to the branching into preterminal portal venules with an inner diameter of $80-40 \mu \mathrm{m}$. This latter portion appears to be the main site of the constrictive response of the portal tree to various constrictive stimuli and, as such, the main mechanism for controlling blood distribution within the liver. Further downstream, the so-called terminal portal venules are endothelial tubes surrounded by 
a thin layer of smooth muscle. These structures do not contract and splits into septal, perilobular, and lobular branches that supply directly blood to sinusoids via inlet venules and give a constant but sluggish blood flow (12). At this last level, a sphincter mechanism is created by the nuclei of endothelial cells residing at origin of sinusoids. The branching of the distribution portion of portal tree is paralleled by the arterial and lymphatic components of the portal tract (13).

\section{Arterial Supply}

Arterial supply, which normally represents from $25 \%$ to $30 \%$ of the hepatic blood flow, satisfies the oxygen demands of the stromal and parenchymal compartments of the liver. The arteries form a peribiliary plexus that surrounds and nourishes small bile ducts. Intrahepatic arteries are thick-walled and become smaller as the arteries branch. Terminal branches contain only endothelium surrounded by a thin adventitia. Entry of arterial blood into sinusoids takes place at different levels, mainly zone 1 and 2 of the acinus. Additional entrances in zone 3 have also been postulated. Drainage of the plexus is both directly in sinusoids and into small branches of the portal veins (14). In physiological conditions, the arterial flow varies inversely with portal vein flow, and compensates for the eventual shortage of portal perfusion $(15,16)$. The hepatic artery provides a pulsatile but small-volume flow that appears to enhance sinusoidal flow, especially in periods of reactive arterial flow, such as the postprandial hyperemia. The proportion of arterial perfusion rises in portal hypertension irrespective of the etiology and reflects a deterioration of liver conditions (17).

\section{The Functional Unit of the Liver-Rappaport's Acinus}

Rappaport's acinus is a parenchymal mass lying between two centrilobular veins. Its axis is a small radicle of the portal triad containing a terminal portal vein (diameter $<40$ $\mu \mathrm{m}$ ), a hepatic arteriole (diameter $>15 \mu \mathrm{m}$ ), nerves, lymph vessels, and bile ducts or cholangioles. This axis, seldom seen by light microscopy, corresponds to the connective tissue septa. Blood drain into centrilobular veins (diameter $<65 \mu \mathrm{m}$ ) from the terminal branch of the portal vein. In sinusoids, flow is unidirectional, from periportal to centrilobular hepatocytes. The concept of functional heterogeneity has been based on this organization. The acinus is arbitrarily divided into three zones; (zone 1) periportal; (zone 2) mediolobular; (zone 3) centrolobular (18). In Rappaport's acinus, blood flows unidirectionally from zone 1 to zone 3. This is because of: (1) the presence of sinusoidal inlet and outlet sphincters composed of sinusoidal lining cells bulging into the lumen; (2) transient leucocyte plugging; (3) variations in the morphology of sinusoids in the different zones; and (4) the contribution of arterial flow at the beginning of the sinusoidal structures (19).

\section{Sinusoids}

Between the genuinely interdigitating networks of afferent and efferent vessels, there is a space filled with plates and columns of hepatocytes, among which a complex network of sinusoids is found. In other words, the hepatic sinusoids can be seen as conduits connecting the terminal portal venule and terminal hepatic arteriole with the hepatic venules. The length of a human sinusoid varies between 223 and $477 \mu \mathrm{m}$. The average velocity of erythrocyte flow in sinusoids ranges between 270 and $410 \mu \mathrm{m} / \mathrm{s}$. Average blood pressure has been measured to be about $4.8 \mathrm{mmHg}$ in terminal portal branches, $30-35 \mathrm{mmHg}$ in arterial blood, and $1.7 \mathrm{mmHg}$ in collecting vessels (20). The huge cross-sectional area 
of the sinusoids is responsible for the normally low transsinusoidal vessel resistance, pressure gradient, and flow velocity. It is estimated that $80 \%$ of the sinusoidal profile would have to be obliterated to cause portal pressure to rise (21). This portion of the liver circulation is unique in comparison to most other capillary beds. Its endothelial lining is made up of flat, lobulated, fenestrated cells, which overlap loosely without being attached to one another, i.e., intercellular junctions are absent. In contrast with other capillaries, hepatic sinusoids are not provided with a basal membrane. The presence of elements of the cytoskeleton in these fenestrations have raised the issue of whether fenestrations may contract and to what extent this may influence the passage of solutes into the space of Disse. This is a space located between the sinusoidal domain of the hepatocyte plasma membrane and the endothelial cells forming the walls of the hepatic sinusoids.

This space, which is not normally discernible in biopsy material by standard light microscopy, is characterized by the presence of different components of the extracellular matrix (different type of collagens mainly type III, but also types I and IV, proteoglycans, laminin, and fibronectin). Fibrillar collagens such as collagen types I and III are orderly distributed to form a supporting framework, whereas other components such as collagen type IV, proteoglycans, and laminin are distributed in order to form a matrix allowing the exchange of macromolecules between the sinusoidal blood and the hepatocytes. The caliber of the sinusoids is variable, typically $6-30 \mu \mathrm{m}$, but can increase up to $180 \mu \mathrm{m}$. Periportal sinusoids are narrow and tortuous facilitating solute-capillary wall interaction, whereas perivenous sinusoids are straighter and wider. The changes of the caliber seem to be mostly passive, depending on regional flow and volume changes but depends also on active contraction of HSC, and changes in the diameter of sinusoidal fenestrations as it will be further expanded.

There are two types of sphincter-like structures at the entering sites of hepatic sinusoids. One is located at the junction between the terminal portal venule and the sinusoid, and is characterized by the large endothelial cells surrounded with Ito cells (HSCs). The other is located at the junction between the terminal hepatic arteriole and the sinusoid, and corresponds to the precapillary sphincter because our enzymohistochemical demonstration of arterial capillaries in close association with the sinusoids combined with intravital microscopy has revealed that the terminal hepatic arteriole directly terminates in the sinusoid.

\section{Control of the Intrahepatic Circulation}

Under physiological conditions, the liver itself is the main site of resistance to portal flow. It should be emphasized that there is no precise mechanism regulating portal flow into the liver and, in normal conditions, the portal tree is able to accept any amount of blood coming from the splanchnic area. In other words, the liver is not able to control the volume of the portal flow, which is mainly determined by resistance vessels of splanchnic organs that drain into the portal venous system. The principal site of resistance within normal hepatic tissue is still a matter of controversy. There are indeed several structures potentially affecting intrahepatic resistance. These include terminal hepatic venules, small portal venules, and, at the sinusoidal level, the state of tension developed by HSC around sinusoids and the number and diameter of sinusoidal fenestrations. However, an active sinusoidal control of the perfusion still represents a controversial issue, as will be further expanded. Arterial inflow, on the other hand, is a subject of clear and effective control, depending mainly on the actual needs of the liver tissue (22). In addition, compensatory relationships exist between the venous and arterial inflows (23), so arterial flow increases 
when portal flow decreases. This occurs as a result of communications among main vessels, sinusoids, and peribiliary venules (24) that open in response to nervous and soluble factors (25). In these cases, portal flow blockade has repercussions on the entire territory downstream from the obstruction, following portal dichotomy and producing an arterial reaction strictly confined to that territory and, therefore, with a sectorial, triangular shape. Moreover, in case of bile duct dilatation, a collapse of the peribiliary plexus that surrounds biliary tree like a meshwork and lacks muscular walls is observed. Because this plexus provides an additional flow of portal blood toward the sinusoids, its impairment or failure results in a further decrease in the total amount of portal blood reaching the sinusoids and increase in arterial inflow of these structures.

\section{BIOLOGY OF PORTAL CELLS INCLUDING SINUSOIDAL ENDOTHELIAL CELLS AND HSCs}

\section{Sinusoidal Endothelial Cells (SECs)}

Liver sinusoidal endothelial cells (SEC) form a continuous lining of the liver capillaries, or sinusoids, separating parenchymal cells and HSC from sinusoidal blood. SEC differ in fine structure from endothelial cells lining larger blood vessels and from other capillary endothelia in that they lack a distinct basement membrane and also contain open pores, or fenestrae, in the thin cytoplasmic projections that constitute the sinusoidal wall (26). This distinctive morphology supports the protective role played by liver endothelium, the cells forming a general barrier against pathogenic agents and serving as a selective sieve for substances passing from the blood to parenchymal and $\mathrm{HSC}$, and vice versa. Another functional characteristic of SEC is their high endocytotic capacity. This function is reflected by the presence of numerous endocytotic vesicles and by the effective uptake of a wide variety of substances from the blood by receptor-mediated endocytosis (27). This capacity, together with the presence of fenestrae and the absence of a regular basal lamina, makes these cells different and unique from any other type of endothelial cell in the body. Accordingly, SEC can be regarded as a "scavenger system," which clears the blood from many different macromolecular waste products that originate from turnover processes in different tissues.

Beside endocytosis, endothelial transport in the liver sinusoidal endothelium occurs through fenestrae without a diaphragm. During this process, the endosomal and lysosomal compartments are bypassed. The exchange of fluids, solutes, and particles is bidirectional, allowing an intensive interaction between the sinusoidal blood and the microvillus surface of the parenchymal cells. Endothelial fenestrae measure between 150 and 175 $\mathrm{nm}$ in transmission electron microscopic preparations, occur at a frequency of 9-13 per $\mu \mathrm{m}^{2}$, and occupy about $10 \%$ of the wall surface. They are large in zone 1 and smaller but more numerous in zone 3 . Endothelial fenestrae do not obstruct most plasmatic macromolecules and enable the exchange of free water and substance within the sinusoids at quite low hydrostatic pressure $(2-3 \mathrm{mmHg})$.

Fenestrae are dynamic structures whose diameter and number vary in response to a variety of hormones, drugs, toxins, diseases, or even to changes in the underlying extracellular matrix. Structural integrity of the fenestrated sinusoidal liver endothelium is essential for the maintenance of a normal exchange of fluids, solutes, particles, and metabolites between the hepatocytes and sinusoidal blood. Changes in the structure and function of fenestrae can have adverse effects on hepatocytes and liver function in general (28). 
Sinusoidal fenestrations can change in size in response to various stimuli, including blood pressure, neural impulses, serotonin, endotoxin, alcohol, and nicotine.

Several studies have explored the mechanisms whereby hormones and cytoskeletalaltering drugs change the fenestral diameter and number. From these studies it became clear that drugs which alter the calcium concentration within SEC also change the fenestrae diameter (29). Recent data indicate that both $\mathrm{Ca}^{2+} \mathrm{Mg}^{2+}$-ATPase and $\mathrm{Ca}^{2+}$ pumpATPase demonstrated on the SEC plasma membrane may be involved in the regulation of intracytoplasmic $\mathrm{Ca}^{2+}$ concentration (30). Other studies have shown that drugs which interfere with the SEC-cytoskeleton mainly alter the number of fenestrae (31). Finally, peculiar reports appeared describing fenestral dynamics in various pathological conditions of the liver, such as hypoxia, increasing venous pressure, irradiation, cold storage, and invasion of the liver by metastatic tumor cells or viruses (26).

Some aspects of SEC function may have relevant implications for the regulation of sinusoidal pressure. The filtration of lipoproteins by open pores is the simplest mechanism for steric selection. However, the fenestrae limit the free access to the parenchymal cell by a factor of 10 . To overcome the difficulty of bringing solutes and (lipid) particles into the space of Disse and in contact with the parenchymal cells, the mechanisms of "forced sieving" and "endothelial massage" has been postulated (28). The hypothesis of "forced sieving" is based on the consideration that red blood cells unilaterally restrict the space in which lipoproteins move in Brownian motion. Red blood cells therefore increase the chance that lipoprotein droplets will escape through the fenestrae. Taking into account that red blood cells pass by in endless numbers while gently touching the fenestrated lining and, in the meantime, constantly adapting their shape to the dimensions of the sinusoid, it is assumed that red blood cells in their turn exert an important effect on the passage of any molecule larger than water through the liver sieve. According to the hypothesis of endothelial massage, white blood cells plug the sinusoid because they have an average size of $8.5 \mu \mathrm{m}$ and, therefore, do not fit into a sinusoid, which measures from $5.9 \mu \mathrm{m}$ in the portal region to $7.1 \mu \mathrm{m}$ in the centrilobular region. In addition, white blood cells are less plastic than other blood cells and do not easily adapt to obstacles or diameter changes of sinusoids. As a result, white blood cells distend the fenestrated endothelium and the space of Disse. As a consequence, fluid in the space of Disse is pushed downstream and when fenestrae are encountered, fluid will be flushed out of Disse's space. After passage of the white blood cells, the space of Disse resumes its original shape, which causes a suction of fresh fluids into the space. In this way, the homeostasis of sinusoidal pressure is maintained by dispersion of lateral force throughout the sinusoidal sieve. It is implicit that a consistent loss of fenestrations, as it is observed in capillarized sinusoids, represents per se, an initial cause of deregulation of this homeostasis and of increased portal pressure.

\section{HSC}

HSC are located in the space of Disse in close contact with hepatocytes and SEC. In human liver, HSC are disposed along the sinusoids with a nucleus-to-nucleus distance of $40 \mu \mathrm{m}$, indicating that the sinusoids are equipped with HSC at certain fixed distances (32). These observations suggest that, although the total number of HSC constitutes a small percentage of the total number of liver cells (approx 5-8\%), their spatial disposition and spatial extension may be sufficient to cover the entire hepatic sinusoidal microcirculatory network. The most evident ultrastructural feature of HSC in normal adult 
Table 1

Action of Vasoactive Agents on Hepatic Stellate Cells

\begin{tabular}{lccc}
\hline Agent & Contraction & Relaxation & {$\left[\mathrm{Ca}^{2+}\right]$ i increase } \\
\hline Endothelin-1 & ++++ & & Coupled \\
Thrombin & ++++ & & Coupled \\
Angiotensin-II & +++ & & Coupled \\
Substance P & +++ & & \\
Adenosine & +++ & & Coupled \\
Thromboxane & +++ & & Coupled \\
Vasopressin & ++++ & & Coupled \\
Platelet-activating factor & + & & Coupled \\
Cysteinyl leukotrienes & +++ & ++ & $*$ \\
Adrenomedullin & & ++ & $*$ \\
Nitric oxide & & +++ & $*$ \\
cAMP increasing agents & & ++ & $*$ \\
Lipo PGE & & +++ & $*$ \\
Atrial natriuretic peptide & & +++ & \\
C-type natriuretic peptide & & & \\
\hline
\end{tabular}

*Relaxation associated with an inhibition of vasoconstrictor induced-[Ca $\left.{ }^{2+}\right]_{\mathrm{i}}$ increase.

liver is the presence of cytoplasmic lipid droplets ranging in diameter from 1 to $2 \mu \mathrm{m}$ (i.e., "fat-storing cells" or "lipocytes") (32). These lipid droplets are involved in the hepatic storage of retinyl esters because of the key role of HSC in the metabolism and storage of retinoids.

The role of HSC in the progression of liver fibrosis has extensively been characterized. As a consequence of chronic liver tissue damage, HSC, as well as other extracellular matrix-producing cells (e.g., fibroblasts and myofibroblasts constitutively present in the portal tract), undergo a process of activation that leads to a phenotype characterized by increased proliferative, motile, and contractile attitudes.

The recognition that HSC are provided with contractile properties represents a key acquisition in the knowledge of the biology of this cell type (33). Contraction of activated HSC occurs in vitro in response to different vasoconstrictors (Table 1). However, this experimental evidence is likely to be more representative of HSC contractile status in fibrotic liver, where contraction of activated HSC in response to various stimuli may have important implications in the pathogenesis of portal hypertension and in the contraction of mature scar tissue. Following two studies published in 1992 (34,35) demonstrating the contraction of HSC in response to different vasoconstrictors, the potential involvement of this cell type in the genesis and progression of portal hypertension has been postulated. Regardless, the potential consequences of the contractile attitude of HSC are still a matter of controversy and some key questions should be addressed before reaching superficial conclusions. These include:

1. Do HSC play a role in the regulation of sinusoidal tone in normal liver?

2. Do HSC influence portal pressure in conditions of developing fibrosis and "capillarization" of sinusoids?

3. Do HSC influence portal pressure in cirrhotic liver? 
Because of their anatomical location, ultrastructural features, and similarities with pericytes regulating blood flow in other organs, HSC have been proposed to function as liver-specific pericytes. As already introduced, branches of the autonomic nerve fibers coursing through the space of Disse show a contact surface with HSC (36), and nerve endings containing substance $\mathrm{P}$ and vasoactive intestinal peptide have been demonstrated in the vicinity of HSC (37). In both a normal and fibrotic liver, the expression of N-CAM, a typical central nervous system adhesion molecule detected in hepatic nerves, and the expression of glial fibrillary acidic protein (GFAP) are restricted, among liver cell types, to HSC (38). These observations, although reinforcing a potential functional relationship between the autonomic nervous system and HSC, raise a current key issue concerning the origin of this cell type, previously considered to be of myogenic origin because of the expression of desmin and smooth muscle $\alpha$-actin ( $\alpha$-SMA). Along these lines, activated HSC express nestin, a class VI intermediate filament protein originally identified as a marker for neural stem cells (39). Remarkably, the expression of this cell marker appears to be restricted to HSC and pericytes of brain parenchyma vessels, among all organspecific pericytes. Another neuroendocrine marker suggesting a combination of mesenchymal and neural/neuroendocrine features in HSC is synaptophysin, a protein involved in neurotransmitter exocytosis. Synaptophysin reactivity is present in perisinusoidal stellate cells in both human and rat normal liver biopsies and the number of synaptophysinreactive perisinusoidal cells is increased in pathological conditions (40). Recent experimental evidence indicates that rat and human HSC express neurotrophins [including nerve growth factor (NGF), brain-derived neurotrophin, neurotrophin 3, and neurotrophin 4/5] and neurotrophin receptors (41). This information cannot be advocated to support the possible neural/neuroendocrine differentiation of HSC because neurotrophins and the relative receptors have been identified in a variety of mesenchymal cells, such as fibroblasts and myofibroblasts, both in normal tissues and in tissues undergoing acute or chronic wound repair. Expression of neurotrophins in tissues other than the central or peripheral nervous system has classically been considered to be aimed at stimulation of outgrowth and maintenance of the peripheral nervous system. However, an increasing number of experimental reports indicate that the neurotrophin/neurotrophin receptor systems is likely implicated in biological events such as cell differentiation, proliferation, survival, and motility. In addition, a significant positive correlation between NGF synthesis e cell contractility, possibly related to the regulation of intracellular calcium homeostasis, has been reported in vascular smooth muscle cells $(42,43)$. In aggregate, these observations suggest a complex interaction between the pathophysiological role of HSC and the function of the peripheral nervous system.

Although these evidences suggest a role of HSC in the regulation of sinusoidal blood flow in normal liver, this issue is still matter of substantial controversy. From the morphological standpoint, some observations argue against the role of $\mathrm{HSC}$ in the regulation of sinusoidal blood flow (44). First, in their in vivo tridimensional disposition, HSC do not have a stellate form (typical of their aspect in bidimensional culture on plastic) but rather a "spider-like" appearance ("arachnocytes") in respect of their small cell body with a series of radiating and parallel slender processes. According to the authors of these observations, cells with this tridimensional disposition are not likely to be "contraction ready." Additional limitations to effective cell contraction are offered by the spatial limitation of the space of Disse, by the intracytoplasmic presence of lipid droplets that prevent microfilaments from assembly in a long span, and by the ultrastructural evidence of a 
limited development of contractile filaments in quiescent HSC. Regardless, studies evaluating the hepatic microcirculation by intravital microscopy techniques have suggested that HSC could be involved in the regulation of sinusoidal tone in normal liver $(45,46)$. Additional matter of debate is provided by studies aimed at quantitating HSC contraction with techniques able to detect the development of contractile forces in response to vasoconstrictors (47). The results of these studies indicate that the magnitude and kinetics of contraction and relaxation are consistent with the hypothesis that HSC may affect sinusoidal resistance. However, for understandable technical reasons, these data were obtained in rat $\mathrm{HSC}$ in primary culture $7 \mathrm{~d}$ after isolation, when a certain degree of activation in culture has occurred. In conclusion, although HSC could be proposed as liver-specific pericytes in reason of their location, spatial distribution, relationship with the peripheral nervous system, and ultrastructural features, no conclusive evidence is presently available concerning their role in the regulation of sinusoidal blood flow in physiological conditions.

\section{MAJOR SIGNALING PATHWAYS RELEVANT TO ENDOTHELIAL-SMOOTH MUSCLE CELL INTERACTIONS}

Several vasoactive agents have been shown to be effective in modulating activated HSC contractility in culture (see Table 1). The role of two vasoregulatory compounds, namely endothelin 1 (ET-1) and NO, has been particularly highlighted.

\section{Endothelin}

Endothelin-1, a potent vasoactive 21-amino-acid peptide secreted by endothelial as well as other cell types, has been shown to exert a multifunctional role in a variety of tissues and cells (48-50), including the liver. Infusion of ET-1 in the isolated perfused rat liver causes a sustained and dose-dependent increase in portal pressure associated with increased glycogenolysis and oxygen consumption (51-53). ET-1 stimulates glycogenolysis, phosphoinositide turnover, and repetitive, sustained intracellular calcium transients in isolated rat hepatocytes $(54,55)$. Other studies indicate that ET-1 may also have important interactions with liver nonparenchymal cells. Cultured sinusoidal endothelial cells isolated from rat liver have been shown to release ET-1 (56), and preferential binding sites for ET-1 have been identified, both in vivo and in vitro (57,58), on HSC. As previously mentioned, ET-1 induces a dose-dependent increase in intracellular free calcium, coupled with cell contraction in this cell type. Importantly, activated rat and human HSC have been shown to express preproET-1 mRNA $(59,60)$ and to release ET-1 in cell supernatants in response to agonists such as angiotensin II, PDGF, TGF- $\beta$, and ET-1 itself (61), thus raising the possibility of a paracrine and autocrine action of ET-1 (62). ET-1 synthesis in HSC is regulated through modulation of endothelin converting enzyme-1 (ECE1), the enzyme that converts precursor ET-1 to the mature peptide, rather than by modulation of the precursor pre-proET-1 (63). Recent evidence suggests that upregulation of 56- and 62-kDa ECE-1 3'-untranslated region (UTR) mRNA binding proteins occurs in HSC after liver injury and during activation in vitro (64). In addition, transforming growth factor- $\beta 1$, a cytokine integral to the wound healing reaction, stimulates ET-1 production by inducing ECE-1 mRNA stabilization.

Overall, it is increasingly evident that the process of HSC activation and phenotypical modulation is characterized by a close and complex relationship with the ET system. The 
ability to synthesize and release ET-1 is associated with a progressive shift in the relative predominance of $\mathrm{ET}_{\mathrm{A}}$ and $\mathrm{ET}_{\mathrm{B}}$ receptors observed during serial subculture: $\mathrm{ET}_{\mathrm{A}}$ are predominant in the early phases of activation, whereas $\mathrm{ET}_{\mathrm{B}}$ receptors become increasingly more abundant in "myofibroblast-like" cells $(60,65)$. The upregulation of the $\mathrm{ET}_{\mathrm{B}}$ receptor is prevented by the incubation of HSC with retinoic acid during the activation process (66), thus confirming that increased expression of this receptor is part of the phenotypical modulation of HSC toward the "myofibroblast-like" phenotype.

The shift in the relative ET receptor densities may be directed at differentiating the possible paracrine and autocrine effects of ET-1 on HSC during the activation process. Indeed, when HSC are provided with a majority of $\mathrm{ET}_{\mathrm{A}}$ receptors (early phases of activation), stimulation with ET-1 causes a dose-dependent increase in cell growth, ERK activity, and expression of c-fos. These effects, likely related to the activation of the Ras-ERK pathway, are completely blocked by pretreatment with BQ-123, a specific $\mathrm{ET}_{\mathrm{A}}$ receptor antagonist (60), and are in agreement with studies performed in other vascular pericytes such as glomerular mesangial cells (67). Conversely, in later stages of activation, when the number of $\mathrm{ET}_{\mathrm{B}}$ receptors increases, ET-1 appears to induce a prevalent antiproliferative effect linked to the activation of this receptor subtype (68). In this setting, the activation of the $\mathrm{ET}_{\mathrm{B}}$ receptor stimulates the production of prostaglandins, leading to an increase in intracellular cAMP, which in turn reduces the activation of both ERK and JNK (69). In addition, both $\mathrm{cAMP}$ and prostaglandins upregulate $\mathrm{ET}_{\mathrm{B}}$ binding sites, thus suggesting the possibility of a positive-feedback regulatory loop. In addition, recent studies have further defined the action of cAMP on the ET-1 receptor system. Cyclic AMP rapidly desensitizes $\mathrm{ET}_{\mathrm{A}}$ in activated HSC and shifts their ET-1 responsiveness from picomolar to nanomolar concentrations with respect to $\mathrm{Ca}^{(2+)}$ signals and $\mathrm{HSC}$ contraction. $\mathrm{ET}_{\mathrm{A}}$ desensitization also occurs in response to prostaglandin $\mathrm{E}_{2}$, adenosine, or $\mathrm{ET}_{\mathrm{B}}$ stimulation (70).

Concerning the potential involvement of HSC in the development of portal hypertension, it is important to note that, at least in human HSC, ET-1-induced cell contraction occurs at any stage of HSC activation (60). Because HSC contraction is always blocked by $\mathrm{ET}_{\mathrm{A}}$ receptor antagonists and never reproduced by selective $\mathrm{ET}_{\mathrm{B}}$ agonists, it is conceivable that the signaling pathways regulating HSC contraction require the activation of a small number of $\mathrm{ET}_{\mathrm{A}}$ receptors and are somehow divergent from those regulating cell growth.

In aggregate, these observations suggest that ET-1 may act as a potent vasoconstrictor agonist regulating intrahepatic blood flow in cirrhotic liver with a potential role in the pathogenesis of portal hypertension. Along these lines, morphological studies have clearly indicated that ET-1 (both at mRNA and protein levels) is markedly overexpressed in different cellular elements present within cirrhotic liver tissue, and particularly in sinusoidal endothelial and HSC in their activated phenotype located in the sinusoids of the regenerating nodules, at the edges of fibrous septa, and in the ECM embedding neoformed vessels within fibrous bands (60). In addition, clinical studies indicate that a direct relationship exists between ET receptor mRNA abundance and the degree of portal hypertension in cirrhotic patients (71).

\section{NO}

NO is a small, relatively stable, free-radical gas that readily diffuses into cells and membranes where it reacts with molecular targets (72). It is important to note that the precise biochemical reactions, which are realized in any biological setting, depend on the concen- 
tration of NO achieved and often on subtle variations in the composition of the intra- and extracellular milieu. Accordingly, the biological actions of NO are often defined as a "double-edged sword." NO may act as a key signaling molecule in physiological processes as diverse as host defense, neuronal communication, and regulation of vascular tone. On the other hand, excessive or not adequately regulated NO synthesis has been implicated as causal or contributing to several pathophysiological conditions including vascular shock, diabetes, and chronic inflammation. Although NO is characterized by a very short half-life, its biochemical interactions with oxyradicals lead to the production of longer-lived compounds such as peroxynitrite, with important local effects. NO is produced from L-arginine by one of the three isoforms of nitric oxide synthase (NOS). The "constitutive" forms of NOS, which respond to changes in intracellular calcium concentration and typically produces small amounts of NO, are expressed by endothelial cells and in neurons, whereas a wide variety of other cells express the "inducible" form of this enzyme, that binds calmodulin at virtually all calcium concentrations and produce remarkably higher amounts of NO. The constitutive forms are regulated by hypoxia, shear stretch, or cytokines, whereas the inducible form is regulated by a large variety of stimuli including cytokines and lipopolysaccharide.

Because the intraportal administration of the NOS inhibitor, $\mathrm{N}^{\omega}$-nitro-L-arginine, increases portal pressure (73), $\mathrm{NO}$ has been postulated to be a regulator of sinusoidal blood flow in normal liver. Along these lines, in vitro and in vivo evidence indicate that SEC express constitutive nitric oxide synthase (eNOS) and produce NO, and increase their production in response to flow (74). However, an endothelial dysfunction associated with a decreased production of $\mathrm{NO}$ in the intrahepatic microcirculation has been documented extensively in cirrhotic liver $(75,76)$, and these defects could directly contribute to the increased intrahepatic resistance typical of portal hypertension. This view is supported by experiments performed in vitro and in animal models by gene transfer of the neuronal NO synthase isoform (nNOS) to sinusoidal endothelial cells or other perisinusoidal cells, such as HSC (77). Expression of nNOS in rat HSC and sinusoidal endothelial cells resulted in increased NO production, and, in HSC, in a reduction of ET-1-induced contractility. Moreover, in two different rat models of cirrhosis and portal hypertension, transduction of livers with recombinant Ad.nNOS significantly reduced intrahepatic resistance and portal pressure.

As in the case of ET-1, circumstantial evidence for a relevance of NO in HSC biology has derived from in vitro studies. Exogenous NO is able not only to prevent ET-1-induced contraction and to relax precontracted cells, but also to reduce the expression of $\alpha$-SMA (78). In addition, interferon- $\gamma$ and other cytokines with or without lipopolysaccharide, as well as hyaluronan fragments induce the expression of the inducible form of NOS and the production of NO in $\mathrm{HSC}(79,80)$. However, at least in human HSC, this effect is very limited and the possibility of an autocrine action of NO in HSC appears merely speculative. In addition to these effects on HSC contraction and contractile proteins, NO has been shown to reduce the expression of procollagen type I mRNA and the secretion of the encoded protein (79). Therefore, it is possible that NO may influence the progression of portal hypertension by reducing the accumulation of fibrillar matrix in key areas such as the fibrous septa, as suggested by evidence deriving from animal models of liver fibrosis (81). It is also conceivable that the reduced synthesis of NO, typical of cirrhotic liver, may further aggravate the fibrogenic progression of the disease and that administration of orally active NO donors could be proposed as a potential antifibrogenic treatment, as 
suggested by recent studies performed in human HSC (82) and in animal models of liver fibrogenesis (83).

Studies employing patch-clamp techniques have provided additional information on the role of membrane ion channels potential relevant for the action of vasoactive agents in HSC. High-conductance $\mathrm{Ca}^{(2+)}$-activated $\mathrm{K}^{(+)}[\mathrm{BK}(\mathrm{Ca})]$ channels modulate the effects of vasoactive factors in contractile cells. This channels were detected in activated human HSC and may modulate the contractile effect of endothelin-1 and mediate the inhibitory action of $\mathrm{NO}$ (84).

\section{Other Vasoactive Agents}

Several studies have evaluated the effects of naturally occurring vasodilators on HSC contractility. These include atrial natriuretic peptide (ANP) (85) and C-type natriuretic peptide (CNP) (86). Both of these agents have been shown to reduce HSC contraction in response by ET-1 or thrombin. In addition, CNP is able to reduce HSC proliferation induced by PDGF-BB (86).

In addition to ET-1, the potential involvement of other vasoconstrictors synthesized and released within liver tissue has been suggested. Titos et al. (87) have reported that in cirrhotic rat liver there is an increased synthesis of cysteinyl leukotrienes (LTs). In this context, hepatocytes exhibit the greatest ability to generate cysteinyl-LTs. It is important to note that these compounds elicit a strong contractile response in activated HSC. These findings further reinforce the concept of an imbalance between vasoconstrictor and vasodilator agents within the intrahepatic circulation of cirrhotic liver. Importantly, the concentration of vasoconstrictors acting on the intrahepatic microvasculature of cirrhotic liver may increase as a consequence of clinical or subclinical events such as infections in the peritoneal cavity, which are clearly associated with a worsening of portal hypertension and with an increased incidence of variceal bleeding (88). This possibility as well other clinical possibilities, including the correct use of the drugs currently indicated in the treatment of portal hypertension (89), should be carefully reconsidered in light of the current knowledge on the cellular and molecular mechanisms of portal hypertension.

\section{REFERENCES}

1. Battaglia DM, Wanless IR, Brady AP, Mackenzie RL. Intrahepatic sequestered segment of liver presenting as focal fatty change. Am J Gastroenterol 1995;90:2238-2239.

2. Madding GF, Kennedy PA. Trauma of the liver. In: Calne RY's Liver Surgery with Operative Color Illustrations. WB Saunders, Philadelphia, PA, 1982, p. 5.

3. Douglas BE, Baggenstoss AH, Hollinshead WH. The anatomy of the portal vein and its tributaries. Surg Gynecol Obstet 1979;91:562-576.

4. Bosch J, Navasa M, Garcia-Pagán JC, De Locy AM, Rodes J. Portal hypertension. Med Clin North Am 1989;73:931-953.

5. Popper H, Elias H, Petty DE. Vascular pattern of the cirrhotic liver. Am J Clin Pathol 1952;22:717-722.

6. Vianna A, Hayes PC, Moscoso G, et al. Normal venous circulation of the gastresophageal junction. A route to understanding varices. Gastroenterology 1987;93:876-889.

7. Crissinger KD, Granger DN. Gastrointestinal blood flow. In: Yamada T, et al., eds. Textbook of Gastroenterology. Lippincott-Williams \& Wilkins, Philadelphia, PA, 1999, p. 519.

8. Granger DN, Kvietys PR, Korthuis R, Premen AJ. Microcirculation of the intestinal mucosa. In: Wood JD, ed. Handbook of Gastrointestinal Physiology. American Physiological Society, 1989, pp. 1405-1474.

9. Benoit JN, Korthuis RJ, Granger DN, Battarbee HD. Splanchnic hemodynamics in acute and chronic portal hypertension. In: Bonzon A, Blendis LM, eds. Cardiovascular Complications of Liver Disease. CRC, Boca Raton, FL, 1990, p. 179. 
10. Jensen JE, Groszmann RJ. Pathophysiology of portal hypertension. In: Kaplowitz N, ed. Liver and Biliary Diseases. Williams \& Wilkins, Baltimore, 1992, pp. 494-503.

11. Bioulac-Sage P, Lafon ME, Saric J, Balabaud C. Nerves and persinusoidal cells in human liver. J Hepatol 1990;10:105-112.

12. Wanless IR. Physioanatomic consideration. In: Schiff ER, Sorrel MF, Maddrey WC, ed. Schiff's Diseases of the Liver, 8th ed., Lippincott-Raven, Philadelphia, PA, 1999, pp. 18,19.

13. Wisse E, De Zanger RB, Jacobs R, McCuskey RS. Scanning electron microscope observations on the structure of portal veins, sinusoids and central veins in rat liver. Scan Electron Microsc 1983;3: $1441-1452$.

14. Nopanitayo W, Grisham JW, Aghajanian JG, Carso JL. Intrahepatic microcirculation: SEM study of the terminal distribution of the hepatic artery. Scan Electron Microsc 1978;11:837-842.

15. Lautt WW. Relationship between hepatic blood flow and overall metabolism: the hepatic arterial buffer response. Fed Proc 1983;42:1662-1666.

16. Ezzat WR, Lautt WW. Hepatic arterial pressure-flow autoregulation is adenosine mediated. Am J Physiol 1986;252:H836-H845.

17. Shikare SV, Baschir K, Abraham P, Tilve GH. Hepatic perfusion index in portal hypertension of cirrhotic and non cirrhotic aetiologies. Nucl Med Comm 1996;17:520-522.

18. Rappaport AM. The microcirculatory hepatic unit. Microvasc Res 1973;6:212-218.

19. Yamamoto K, Sherman I, Phillips MJ, Fisher MM. Three-dimensional observation of the hepatic arterial terminations in the rat, hamster and human liver by scanning electron microscopy of microvascular casts. Hepatology 1985;5:452-456.

20. Rappaport AM. Physioanatomic consideration. In: Schiff L, Schiff ER, eds. Diseases of the Liver. JB Lippincott, Philadelphia, PA, 1987, pp. 1-46.

21. Sherlock S, Doodley J. The portal venous system and portal hypertension. In: Sherlock S, Doodley J, eds. Disease of the Liver and Biliary System, 7th ed. Blackwell Science, Oxford, UK, 1997.

22. McCuskey RS, Reilly FD. Hepatic microvasculature: dynamic structure and its regulation. Semin Liver Dis 1993;13:1-12.

23. Ternberg JL, Butcher HR Jr. Blood-flow relation between hepatic artery and portal vein. Science 1965; 150:1030,1031.

24. Itai Y, Moss AA, Goldberg HI. Transient hepatic attenuation difference of lobar or segmental distribution detected by dynamic computed tomography. Radiology 1982;144:835-839.

25. Kawasaki T, Carmichael FJ, Saldivia V, Roldan L, Orrego H. Relationship between portal venous and hepatic arterial blood flows. Spectrum of response. Am J Physiol 1990;259:1010-1018.

26. Braet F, Wisse E. Structural and functional aspects of liver sinusoidal endothelial cell fenestrae: a review. Comp Hepatol 2002;1:1-17.

27. De Leeuw AM, Brouwer A, Knook DL. Sinusoidal endothelial cells of the liver: fine structure and function in relation to age. J Electron Microsc Tech 1990;14:218-236.

28. Wisse E, De Zanger RB, Charels K, Van Der Smissen P, McCuskey RS. The liver sieve: considerations concerning the structure and function of endothelial fenestrae, the sinusoidal wall and the space of Disse. Hepatology 1985;5:683-692.

29. Oda M, Kazemoto $\mathrm{S}$, Kaneko $\mathrm{H}$, et al. Involvement of $\mathrm{Ca}^{++}$-calmodulin-actomyosin system in the contractility of hepatic sinusoidal endothelial fenestrae. In: Knook DL, Wisse E, eds. Cells of the Hepatic Sinusoid 4. Kupffer Cell Foundation, Leiden, 1993, pp. 174-178.

30. Yokomori H, Oda M, Ogi M, et al. Hepatic sinusoidal endothelial fenestrae express plasma membrane $\mathrm{Ca}^{++}$pump and $\mathrm{Ca}^{++} \mathrm{Mg}^{++}$-ATPase. Liver 2000;20:458-464.

31. Steffan AM, Gendrault JL, Kirn A. Increase in the number of fenestrae in mouse endothelial liver cells by altering the cytoskeleton with cytochalasin B. Hepatology 1987;7:1230-1238.

32. Wake K. Liver perivascular cells revealed by gold and silver impregnation methods and electron microscopy. In: Motta P, ed. Biopathology of the Liver, an Ultrastructural Approach. Kluwer, Dordrecht, 1988, pp. 23-26.

33. Pinzani M, Gentilini P. Biology of hepatic stellate cells and their possible relevance in the pathogenesis of portal hypertension in cirrhosis. Semin Liver Dis 1999;397-410.

34. Pinzani M, Failli P, Ruocco C, et al. Fat-storing cells as liver-specific pericytes: spatial dynamics of agonist-stimulated intracellular calcium transients. J Clin Invest 1992;90:642-646. 
35. Kawada N, Klein H, Decker K. Eicoesanoid-mediated contractility of hepatic stellate cells. Biochem J 1992;285:367-371.

36. Lafon ME, Bioulac-Sage P, LeBail N. Nerves and perisinusoidal cells in human liver. In: Wisse E, Knook DL, Decker K, eds. Cells of Hepatic Sinusoid. Kupffer Cell Foundation, Riswijk, 1989, pp. 230-234.

37. Ueno T, Inuzuka S, Torimura T, et al. Distribution of substance $P$ and vasoactive intestinal peptide in the human liver. Light and electron immunoperoxidase methods of observation. Am J Gastroenterol 1991;138:1233-1242.

38. Knittel T, Aurisch S, Neubauer K, Eichhorst S, Ramadori G. Cell-type-specific expression of neural cell adhesion molecule (N-CAM) in Ito cells of rat liver. Am J Pathol 1996;149:449-462.

39. Niki T, Pekny M, Hellemans K, et al. Class VI intermediate filament protein nestin is induced during activation of rat hepatic stellate cells. Hepatology 1999;29:520-527.

40. Cassiman D, van Pelt J, De Vos R, et al. Synaptosphysin: a novel marker for human and rat hepatic stellate cells. Am J Pathol 1999;155:1831-1839.

41. Cassiman D, Denef C, Desmet VJ, Roskams T. Human and rat hepatic stellate cells express neurotrophins and neurotrophin receptors. Hepatology 2001;33:148-158.

42. Sherer TB, Neff PS, Hankins GR, Tuttle JB. Mechanisms of increased NGF production in vascular smooth muscle of the spontaneous hypertensive rat. Exp Cell Res 1998;241:186-193.

43. Hasan W, Zhang R, Liu M, Warn D, Smith PG. Coordinate expression of NGF and alpha-smooth muscle actin mRNA and protein in cutaneous wound tissue of developing and adult rats. Cell Tissue Res 2000;300:97-109.

44. Ekataksin W, Kaneda K. Liver microvascular architecture: an insight into the pathophysiology of portal hypertension. Semin Liver Dis 1999;19:359-382.

45. Zhang JX, Pegoli W Jr, Clemens MG. Endothelin-1 induces direct constriction of hepatic sinusoids. Am J Physiol 1994;266:G624-G632.

46. Zhang JX, Bauer M, Clemens MG. Vessel- and target cell-specific actions of endothelin-1 and endothelin-3 in rat liver. Am J Physiol 1995;269:G269-G277.

47. Thimgan MS, Yee HF Jr. Quantitation of rat hepatic stellate cell contraction: stellate cells' contribution to sinusoidal tone. Am J Physiol 1999;277:G137-G143.

48. Yanagisawa M, Masaki T. Endothelin, a novel endothelium-derived peptide. Biochem Pharmacol 1989; 38:1877-1883.

49. Simonson MS, Dunn MJ. Endothelins: a family of regulatory peptides. Hypertension 1991;17:856-863.

50. Simonson MS. Endothelins: multifunctional renal peptides. Physiol Rev 1993;73:375-411.

51. Gandhi CR, Stephenson K, Olson MS. Endothelin, a potent peptide agonist in the liver. J Biol Chem $1990 ; 265: 17,432-17,435$.

52. Roden M, Vierhapper H, Liener K, Waldhausl W. Endothelin-1-stimulated glucose production in vitro in the isolated perfused rat liver. Metabolism 1992;41:290-295.

53. Thran-Thi T-A, Kawada N, Decker K. Regulation of endothelin-1 action on the perfused rat liver. FEBS Lett 1993;318:353-357.

54. Gandhi CR, Behal RH, Harvey SA, Nouchi TA, Olson MS. Hepatic effects of endothelin. Receptor characterization and endothelin-induced signal transduction in hepatocytes. Biochem J 1992;287:897-904.

55. Serradeil-Le Gal C, Jouneaux C, Sanchez-Bueno A, et al. Endothelin action in rat liver. Receptors, free $\mathrm{Ca}^{2+}$ oscillations, and activation of glycogenolysis. J Clin Invest 1991;87:133-138.

56. Rieder H, Ramadori G, Meyer zum Buschenfelde KH. Sinusoidal endothelial liver cells in vitro release endothelin: augmentation by transforming growth factor $\beta$ and Kupffer cell-conditioned media. Klin Wochenschr 1991;69:387-391.

57. Furoya S, Naruse S, Nakayama T, Nokihara K. Binding of ${ }^{125}$ I-endothelin-1 to fat-storing cells in rat liver revealed by electron microscopic radioautography. Anat Embryol 1992;185:97-100.

58. Gondo K, Ueno T, Masaharu S, Sakisaka S, Sata M, Tanikawa K. The endothelin-1 binding site in rat liver tissue: light- and electron-microscopic autoradiographic studies. Gastroenterology 1993;104: $1745-1749$.

59. Housset CN, Rockey DC, Bissel DM. Endothelin receptors in rat liver: lipocytes as a contractile target for endothelin 1. Proc Natl Acad Sci USA 1993;90:9266-9270.

60. Pinzani M, Milani S, DeFranco R, et al. Endothelin 1 is overexpressed in human cirrhotic liver and exerts multiple effects on activated hepatic stellate cells. Gastroenterology 1996;110:534-548. 
61. Rockey DC, Fouassier L, Chung JJ, et al. Cellular localization of endothelin-1 and increased production in liver injury in the rat: potential for autocrine and paracrine effects on stellate cells. Hepatology 1998;27:472-480.

62. Gabriel A, Kuddus RH, Rao AS, Watkins WD, Ghandi CR. Superoxide-induced changes in endothelin (ET) receptors in hepatic stellate cells. J Hepatol 1998;29:614-627.

63. Shao R, Yan W, Rockey DC. Regulation of endothelin-1 synthesis by endothelin-converting enzyme1 during wound healing. J Biol Chem 1999;274:3228-3234.

64. Shao R, Shi Z, Gotwals PJ, Koteliansky VE, George J, Rockey DC. Cell and molecular regulation of endothelin-1 production during hepatic wound healing. Mol Biol Cell 2003;14:2327-2341.

65. Reinehr RM, Kubitz R, Peters-Regehr T, Bode JG, Haussinger D. Activation of rat hepatic stellate cells in culture is associated with increased sensitivity to endothelin 1. Hepatology 1998;28:1566-1577.

66. Chi X, Anselmi K, Watkins S, Gandhi CR. Prevention of cultured rat stellate cell transformation and endothelin-B receptor upregulation by retinoic. Br J Pharmacol 2003;139:765-774.

67. Wang YZ, Pouyssegur J, Dunn MJ. Endothelin stimulates mitogen-activated protein kinase activity in mesangial cells through ET(A). J Am Soc Nephrol 1994;5:1074-1080.

68. Mallat A., Fouassier F, Preaux AM, et al. Growth inhibitory properties of endothelin-1 in human hepatic myofibroblastic Ito cells: an endothelin B receptor-mediated pathway. J Clin Invest 1995;96:42-49.

69. Mallat A, Preaux A-M, Serradeil-Le Gal C, et al. Growth inhibitory properties of endothelin-1 in activated human hepatic stellate cells: a cyclic adenosine monophosphate-mediated pathway. J Clin Invest 1996;98:2771-2778.

70. Reinehr R, Fischer R, Haussinger D. Regulation of endothelin-A receptor sensitivity by cyclic adenosine monophosphate in rat hepatic stellate cells. Hepatology 2002;36:861-873.

71. Leivas A, Jimenez W, Bruix J, et al. Gene expression of endothelin-1 and ET(A) and ET(B) receptors in human cirrhosis: relationship with hepatic hemodynamics. J Vasc Res 1998;35:186-193.

72. Gross SS, Wolin MS. Nitric oxide: pathophysiological mechanisms. Annu Rev Physiol 1995;57: 737-769.

73. Mittal MK, Gupta TK, Lee FY, Sieber CC, Groszmann RJ. Nitric oxide modulates hepatic vascular tone in normal rat liver. Am J Physiol 1994;267:G416-G422.

74. Shah V, Haddad FG, Garcia-Cardena G, et al. Liver sinusoidal endothelial cells are responsible for nitric oxide modulation of resistance in the hepatic sinusoids. J Clin Invest 1997;100:2923-2930.

75. Rockey DC, Chung JJ. Reduced nitric oxide production by endothelial cells in cirrhotic rat liver: endothelial dysfunction in portal hypertension. Gastroenterology 1998;114:344-351.

76. Gupta TK, Toruner M, Chung MK, Groszmann RJ. Endothelial dysfunction and decreased production of nitric oxide in the intrahepatic microcirculation of cirrhotic rats. Hepatology 1998;28:926-931.

77. Yu Q, Shao R, Qian HS, George SE, Rockey DC. Gene transfer of the neuronal NO synthase isoform to cirrhotic rat liver ameliorates portal hypertension. J Clin Invest 2000;105:741-748.

78. Kawada N, Kuroki T, Uoya M, Inoue M, Kobayashi K. Smooth muscle $\alpha$-actin expression in rat hepatic stellate cell is regulated by nitric oxide and cAMP. Biochem Biophys Res Comm 1996;229:238-242.

79. Casini A, Ceni E, Salzano R, et al. Neutrophil-derived superoxide anion induces lipid peroxidation and stimulates collagen synthesis in human hepatic stellate cells. Role of nitric oxide. Hepatology 1997; 25:361-367.

80. Rockey DC, Chung JJ, McKee CM, Noble PW. Stimulation of inducible nitric oxide synthase in rat liver by hyaluronan fragments. Hepatology 1998;27:86-92.

81. Rockey DC, Chung JJ. Regulation of inducible nitric oxide synthase and nitric oxide during hepatic injury and fibrogenesis. Am J Physiol 1997;273:G124-G130.

82. Failli P, DeFranco RMS, Caligiuri A, et al. Nitric oxide-generating vasodilators inhibit platelet-derived growth factor-induced proliferation and migration of activated human hepatic stellate cells. Gastroenterology 2000;119:479-492.

83. Fiorucci S, Antonelli E, Morelli O, et al. NCX-1000, a NO-releasing derivative of ursodeoxycholic acid, selectively delivers $\mathrm{NO}$ to the liver and protects against development of portal hypertension. Proc Natl Acad Sci USA 2001;98:8897-8902.

84. Gasull X, Bataller R, Gines P, et al. Human myofibroblastic hepatic stellate cells express $\mathrm{Ca}(2+)-$ activated $\mathrm{K}(+)$ channels that modulate the effects of endothelin-1 and nitric oxide. J Hepatol 2001;35: $739-748$. 
85. Gorbig MN, Gines P, Bataller R, et al. Atrial natriuretic peptide antagonizes endothelin-induced calcium increase in cultured human hepatic stellate cells. Hepatology 1999;30:501-509.

86. Tao J, Mallat A, Gallois C, et al. Biological effects of C-type natriuretic peptide in human myofibroblastic hepatic stellate cells. J Biol Chem 1999;274:23,761-23,769.

87. Titos E, Claria J, Bataller R, et al. Hepatocyte-derived cysteinyl leukotrienes modulate vascular tone in experimental cirrhosis. Gastroenterology 2000;119:794-805.

88. Goulis J, Patch D, Burroughs AK. Bacterial infection in the pathogenesis of variceal bleeding. Lancet 1999;353:139-142.

89. Wiest R, Tsai M-H, Groszmann R. Octreotide potentiates PKC-dependent vasoconstrictors in portalhypertensive rats. Gastroenterology 2001;120:975-983. 



\section{焦 Springer}

http://www.springer.com/978-1-58829-386-2

Portal Hypertension

Pathobiology, Evaluation, and Treatment

Sanyal, A.J.; Shah, V.H. (Eds.)

2005, 528 p. 126 illus., 1 illus. in color., Hardcover

ISBN: 978-1-58829-386-2

A product of Humana Press 